\title{
QUANTIFICATION OF SPATIAL VARIABILITY FOR TRANSVERSE ELASTIC MODULUS OF SPRUCE WOOD
}

\author{
Alireza Farajzadeh Moshtaghin ${ }^{1}$, Steffen Franke ${ }^{2}$, Thomas Keller ${ }^{1}$, and Anastasios P. \\ Vassilopoulos $^{1}$ \\ ${ }^{1}$ Composite Construction Laboratory (CCLab), \\ Ecole Polytechnique Fédérale de Lausanne \\ Station 16, Bâtiment BP, CH-1015 Lausanne, Switzerland \\ e-mail: alireza.farajzadehmoshtaghin@epfl.ch, thomas.keller@epfl.ch, \\ anastasios.vasilopoulos@epfl.ch \\ ${ }^{2}$ Institute for Timber Construction, Structures and Architecture \\ Bern University of Applied Sciences \\ Solothurnstrasse 102, CH-2500 Biel 6, Switzerland \\ steffen.franke@bfh.ch
}

Keywords: Clear Spruce Wood, Transverse Elastic Modulus, Tensile Tests, Random Spatial Variability, Mesostructure.

\begin{abstract}
Different factors such as age, location of timber within the tree, structural imperfections, load history such as wind and snow etc. can affect the material properties of timber. Consequently, there is a high variability in the mechanical properties which is sometimes referred to as 'random spatial variability'. In this work, the spatial variability of the transverse elastic modulus of clear spruce wood is quantified by conducting mechanical tests. Specimens of $120 \mathrm{~mm}$ nominal length with a small cross-sectional area were prepared and their quasistatic behavior was experimentally investigated under tensile loading. In addition to the global displacement monitoring, the local deformations along the length of each specimen were measured. Using these data, the spatial variability of the elastic modulus was experimentally characterized in both longitudinal and transverse directions. Variability coefficients were defined to quantify the variability in both directions. Also, the effect of the mesostructure of the clear timber on the local elastic modulus was examined. The results show a very significant spatial variability in the transverse elastic modulus, more $1000 \%$ within some specimens. The variability of the transverse elastic modulus is much higher in the transverse direction. The grain angle has the most important effect on the value of the local elastic modulus. The results of this work can be considered as part of establishing advanced stochastic models with ransom spatial variability for timber structures.
\end{abstract}




\section{INTRODUCTION}

Different factors such as age, location of timber within the tree, structural imperfections, load history such as wind and snow etc. can affect the material properties of timber taken from the same species, and grown in the same geographical location and local growth conditions. Consequently, there is a high inherent variability in the timber mechanical properties [1]. This variability is both spatial and random, and is sometimes referred to as 'random spatial variability' $[2,3]$.

A few experimental works have been devoted to the characterization of the spatial variability in the transverse mechanical properties of timber and timber products. Regarding the clear timber, Xavier et al. [4] used the unnotched Iosipescu test for the study of the radial variability of stiffness parameters of maritime pine wood. Pereira et al. [5] conducted tensile tests on cubic specimens cut in different radial positions and heights of Pinus pinaster tree. The results show that the radial transverse modulus and the shear transverse modulus decrease from tree center outwards. Also, Brandner and Schickhofer [6] used EN standard-type specimen (cubic bulk material) to investigate the spatial correlation in the transverse tensile strength and elastic modulus along the tree stem.

Nevertheless, the characterization of spatial variability in the transverse local elastic modulus at mesoscale (a few millimeters) has not been studied in detail in the literature. Consequently, the spatial variability is frequently neglected in the probabilistic simulations of timber structures [7-9]. The knowledge of the random spatial variability in elastic parameters can lead to predicting more accurate stress fields within the material. This can improve structural designs with a required reliability levels.

The mesostructure of clear wood is mainly characterized by earlywood-latewood arrangements. Concerning the transverse plane, the mechanical properties are superior in the radial direction [10]. Therefore, when the latewood are locally perpendicular to the nominal axis of a specimen, which is cut in the transverse plane, the local mechanical properties are higher.

Specimens of $120 \mathrm{~mm}$ lengths were cut in the transverse direction of clear spruce wood and their quasi-static behavior was experimentally investigated under tensile loading. In addition to the global displacement monitoring, the local deformations along the length of each specimen were measured. The spatial variability of the elastic modulus was experimentally characterized. The effect of the mesostructure of the clear timber on the local elastic modulus was examined.

\section{EXPERIMNETAL INVESTIGATION}

Clear Norway spruce wood was used for the specimens' preparation in this study. All specimens were conditioned to $12 \%$ moisture content and were tested at the laboratory temperature of $22 \pm 3 \mathrm{C}^{\circ}$. Three boards were cut from the same batch of spruce wood in the radiallongitudinal plane. These were used for cutting regularly positioned transverse specimens and designated as REB1, REB2 and REB3. Figure 1 shows REB1.

The nominal length of the specimens was $120 \mathrm{~mm}$ with a square cross section of $4 \times 4 \mathrm{~mm}^{2}$. A total of 52 specimens were tested on a $5 \mathrm{kN}$ electromechanical Walter+Bai testing machine. Quasi-static tensile tests were performed in displacement-control mode. A stroke rate of 2 $\mathrm{mm} / \mathrm{min}$ for the specimens was used on the basis of previous preliminary experiments so that the final failure occurred within $180 \pm 60 \mathrm{~s}$ throughout the testing program.

A video extensometer system composed of a 10-bit Sony XCLU1000 CCD connected to a Fujinon HF35SA-1, $35 \mathrm{~mm}$, f 1.4-22 lens with an accuracy of $\pm 0.005 \mathrm{~mm}$ was used during the experiments to measure the axial deformation. Prior to the tests, black target dots of $1.1 \mathrm{~mm}$ diameter were applied on the specimen surfaces. The distance between each two con- 
secutive dots was $4 \mathrm{~mm}$. A typical specimen mounted in the testing rig is shown in Fig. 2. The axial coordinates of the dots were recorded at a frequency of $5 \mathrm{~Hz}$ by the video extensometer throughout loading. Using these data, the engineering strain between each two consecutive dots was calculated, designated as the local strain. These data were used for calculation of the local elastic modulus. Also, using the displacements of the first and last dots on each specimen, an overall strain for each nominal length was obtained. These data were used for calculating the effective elastic modulus, for each specimen. Nominal axial stresses were calculated by using the load measurements and the initial cross-sectional areas.

\section{SPATIAL VARIABILITY IN THE MECHANICAL PROPERTIES}

The spatial variability of the transverse elastic modulus along the transverse direction are shown in Fig. 3 for the three REBs. The three boards are different in terms of local elastic modulus variations. On average, the third board is stiffer than the second board and the second board is stiffer than the first board. In most specimens cut from these boards, the local modulus value gradually increases from the one side, reaches a maximum value somewhere in the middle, and then decreases. Also, the scatter in the results is more significant in the second

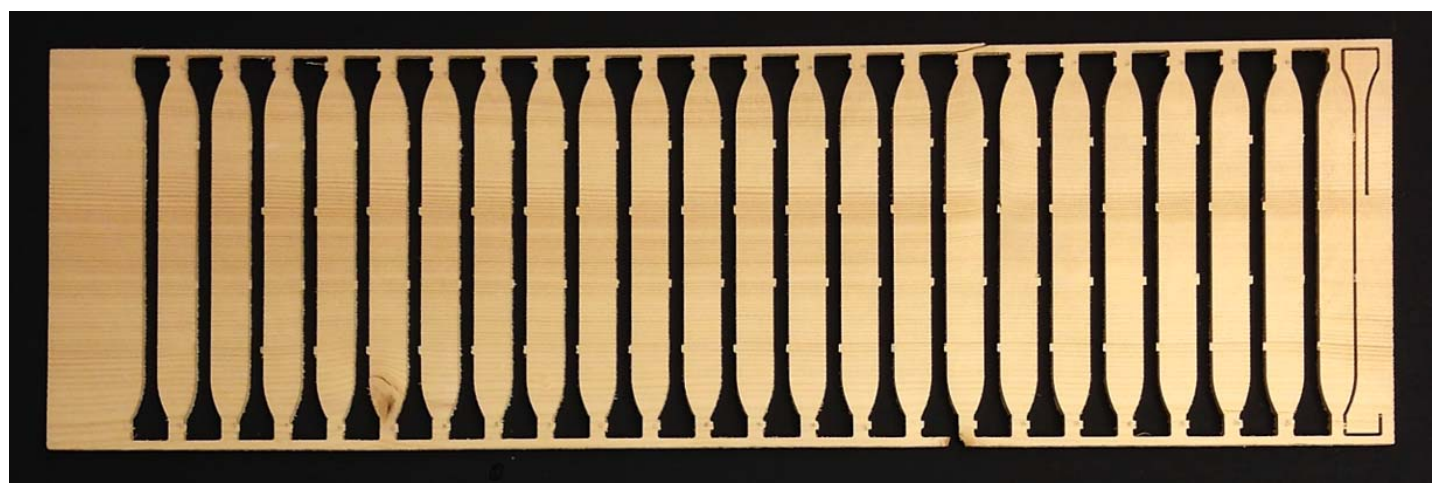

Figure 1: Board REB1 with regular arrangement of transverse specimens.

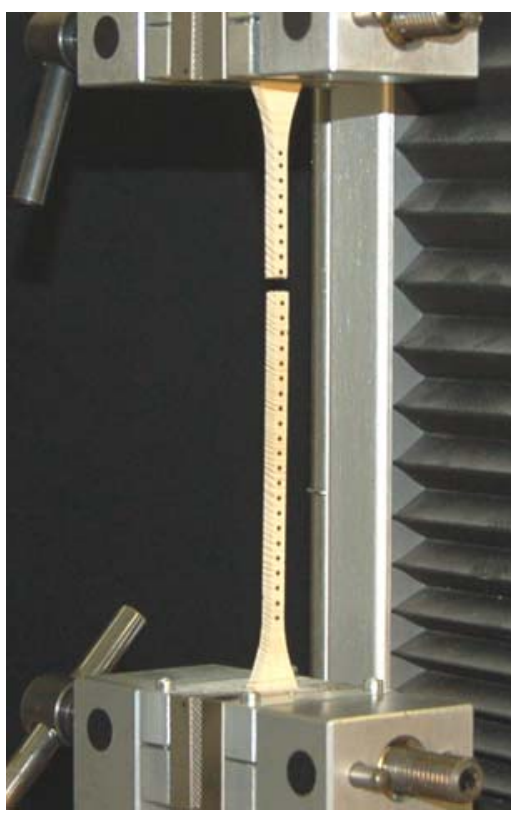

Figure 2: Specimen of $120 \mathrm{~mm}$ nominal length with applied dots inside machine grips.

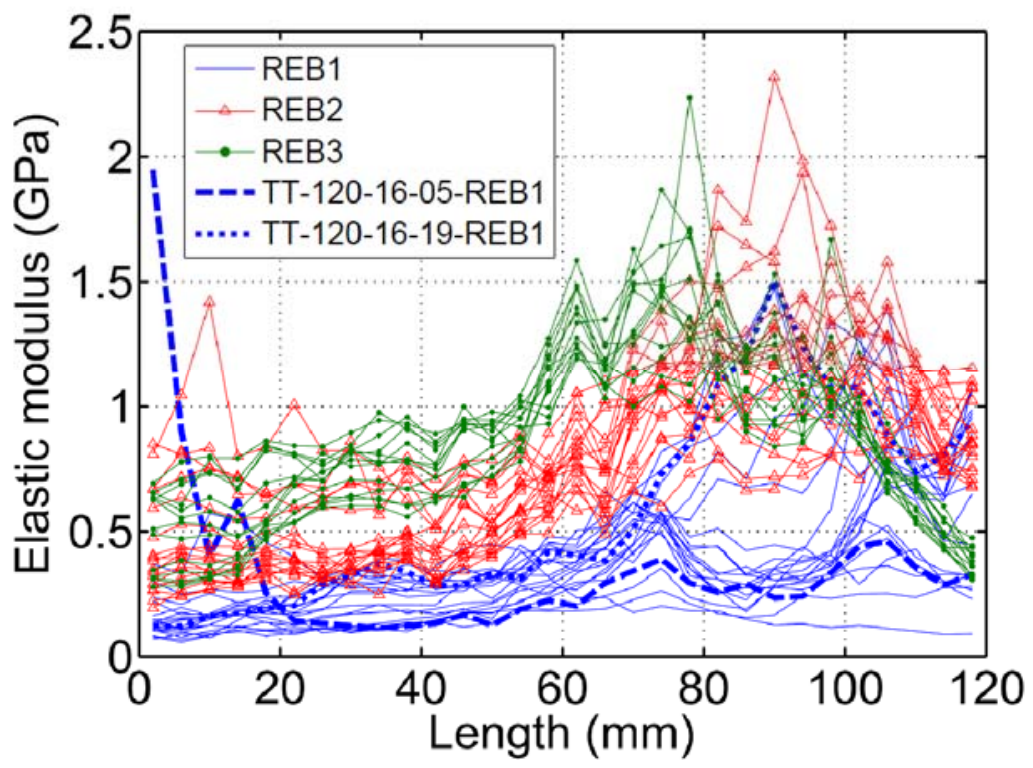

Figure 3: Spatial variability of the transverse elastic modulus in the transverse direction in 3 REBs. 
board compared to the other two boards. There are major changes in the trend of the modulus variability in the first board in a few specimens compared to the others such as the highlighted dashed and dotted curves for specimens TT-120-16-05-REB1 (TT is transverse tensile, 120 is length, 16 is cross section, 05 is specimen number and REB1 is the board name) and TT-12016-19-REB1, respectively, in Fig. 3. In the first case, first four $4 \mathrm{~mm}$ segments or the specimen were affected by the presence of a small nearby knot, which increased the local modulus very significantly. In the second case, the presence of a small knot in the board changed the mesostructure of the specimens 16 to 21 in REB1 at the range between $70 \mathrm{~mm}$ to $120 \mathrm{~mm}$.

The variability of the local elastic modulus due to changing the spatial position within individual specimens (within-specimen variability or WSV) and its variability due to switching between specimens (between-specimen variability or BSV) contribute to the total variability of the local elastic modulus. In Table 1, the COVs for total variability of the local elastic modulus, the average COV for WSV and the COV for BSV are given. It is seen that the contribution of the WSV is higher than that of the BSV.

Variability of the transverse modulus in the longitudinal direction in the three REBs is plotted in Figs. 4-6. In each board, 30 paths longitudinal paths exist. Four curves have been highlighted in each figure in order to show how the longitudinal variability of the transverse modulus differs from lower paths to upper paths. On average, the longitudinal variability is higher in the first board.

\begin{tabular}{lll}
\hline Parameter & Data set size & COV $(\%)$ \\
\hline Local modulus (total variability) & 1560 & 60.65 \\
Local modulus of individual specimens (WSV) & 30 (each specimen) & 48.02 (average) \\
Effective modulus (BSV) & 52 & 44.06 \\
\hline
\end{tabular}

Table 1: Variability of local and effective elastic moduli of $120 \mathrm{~mm}$ specimens.

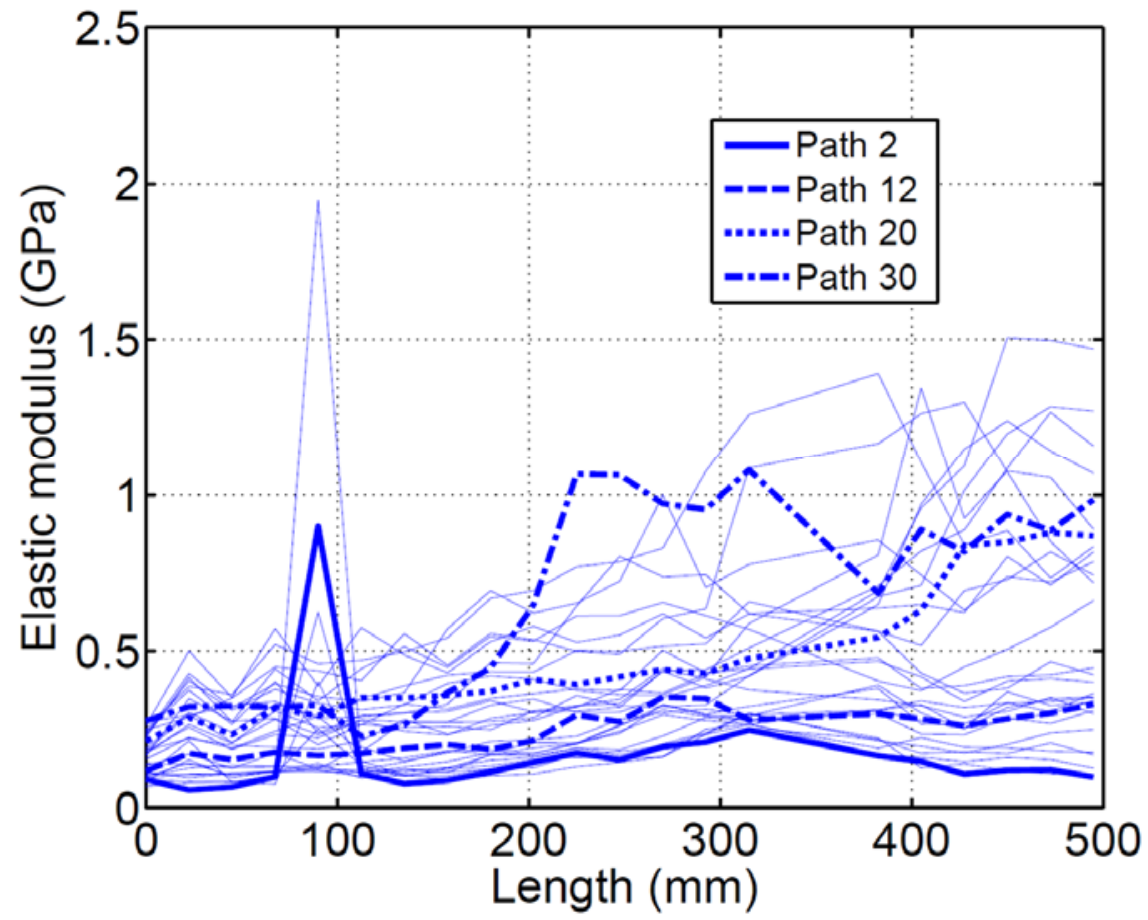

Figure 4: Spatial variability of the transverse elastic modulus in the longitudinal direction in REB1 


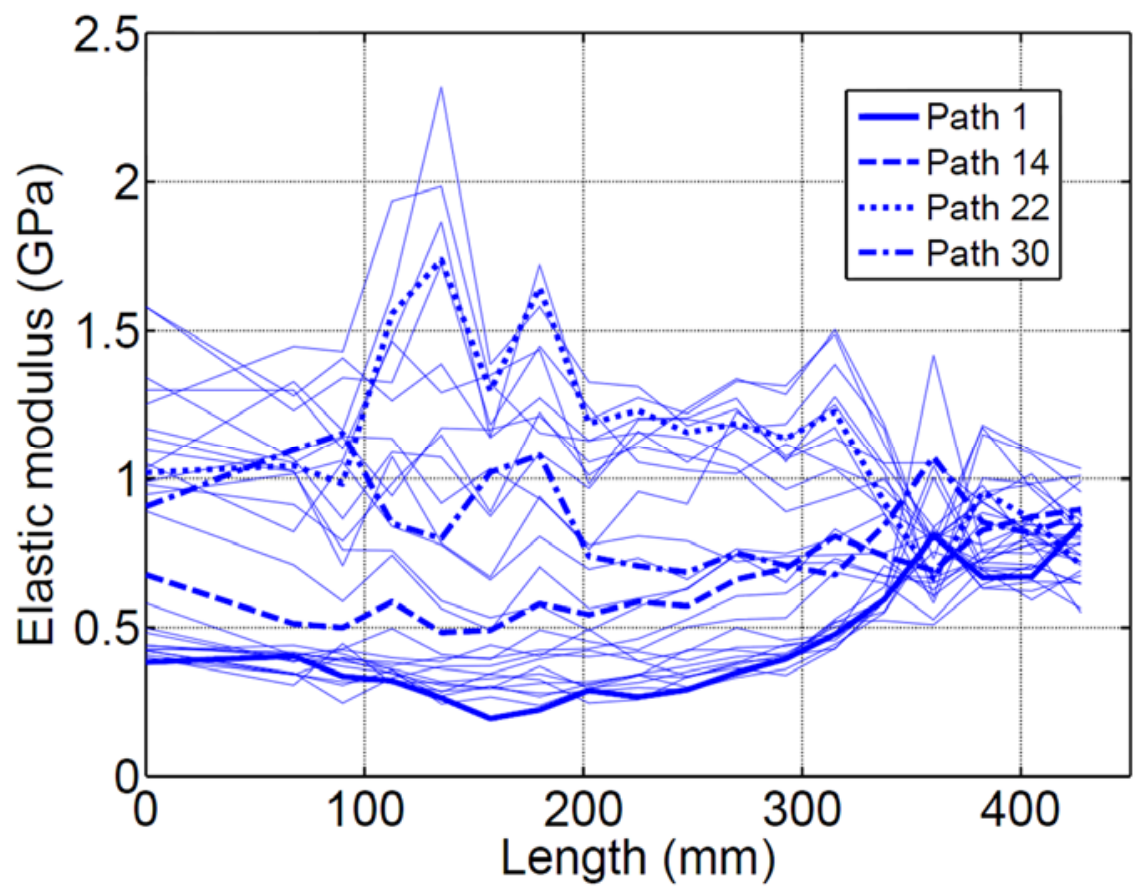

Figure 5: Spatial variability of the transverse elastic modulus in the longitudinal direction in REB2

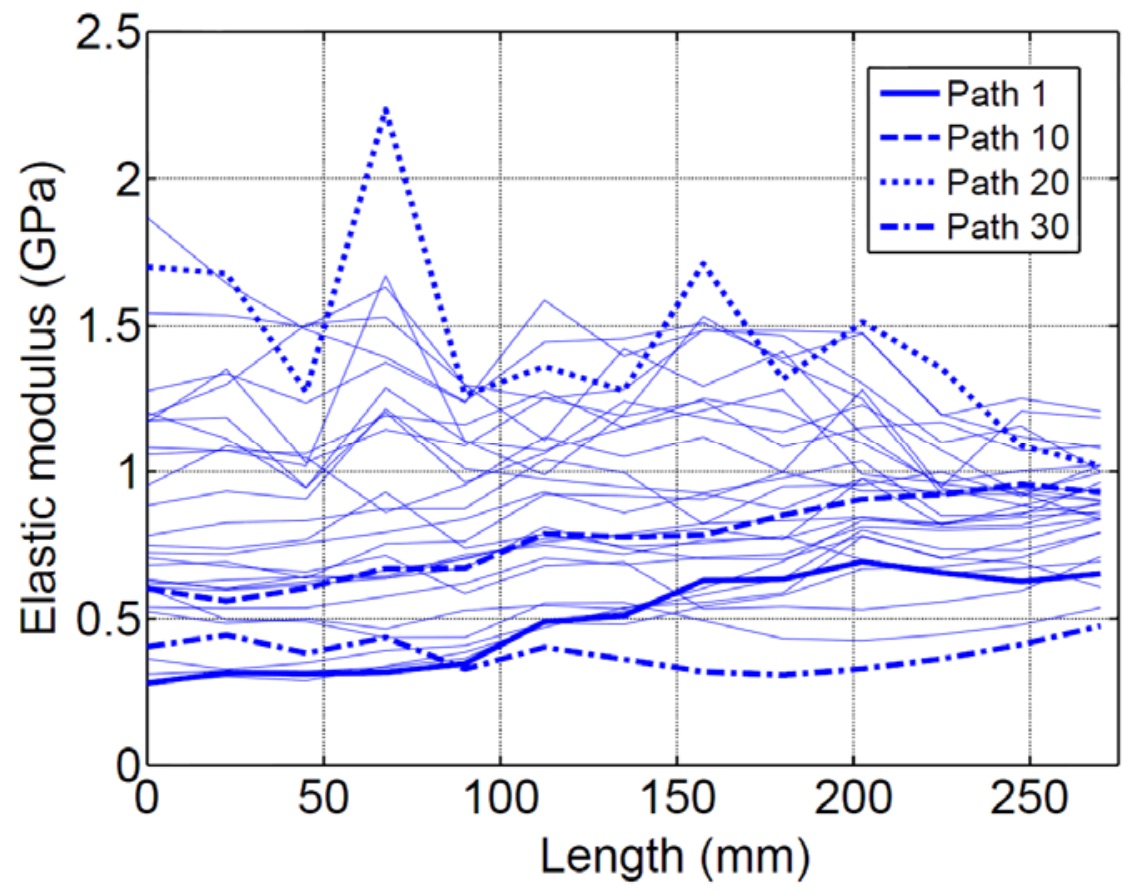

Figure 6: Spatial variability of the transverse elastic modulus in the longitudinal direction in REB3

As a general trend in REB1, the upper paths have higher values of the modulus compared to the lower paths. The paths are closer to each other in the left half length (lower scatter) of the board compared to the right half length (higher scatter). The unusual increase in the value of the transverse modulus, at the longitudinal position of $90 \mathrm{~mm}$, as mentioned above for specimen TT-120-16-05-REB1, is due to a small nearby knot. Excluding this unusual variation at $90 \mathrm{~mm}$ position, the local modulus variation within individual paths ranges from 183.4 $\mathrm{MPa}$ to $1340.5 \mathrm{MPa}$, with a mean value of $581.3 \mathrm{MPa}$. The corresponding values for the 
transverse paths in this board are 208.9, 1372.8 and 869.0 MPa. The mean variation in the longitudinal direction is significantly lower than in the transverse direction.

In the second board, the upper paths have again higher values. The difference between paths after the position of $320 \mathrm{~mm}$ from the left side is considerably lower than before that position. The minimum and maximum variation in the individual paths is $394.1 \mathrm{MPa}$ and 1650.8 MPa, with a mean value of $686.4 \mathrm{MPa}$. These values for transverse paths are 484.0 $\mathrm{MPa}, 2075.5 \mathrm{MPa}$ and $1064.5 \mathrm{MPa}$, respectively. Again, the mean variation in the transverse direction is higher than in the longitudinal direction.

In the third board, the longitudinal variability of the transverse elastic modulus is more uniformly distributed, although the scatter is slightly reduced from left to right. Generally, middle paths are stiffer. In this case, the minimum, the maximum and the mean variation in individual paths are 167.0 MPa, 1216.1 MPa and 421.9 MPa, respectively, which are considerably lower than the corresponding values for the transverse paths, namely $733.6 \mathrm{MPa}$, 1918.5 MPa and 1205.4 MPa. Similar to previous cases, the mean variation in the transverse direction is significantly higher than in the longitudinal direction.

The spatial variations of the elastic modulus in the three boards are shown in Fig. 7 as $2 \mathrm{D}$ contours. In order to compare the degree of the variability in the boards, three coefficients are defined for each board. The first is the ratio between the mean variation in the transverse direction and the transverse distance, the second is the ratio between the mean variation in the longitudinal direction and the longitudinal distance and the third is the ratio of these two coefficients, designated as anisotropy ratio, in the sense of different levels of variation in different directions. These values are provided in Table 2. It can be seen that the longitudinal variability coefficients are much lower than the transverse variability coefficient.

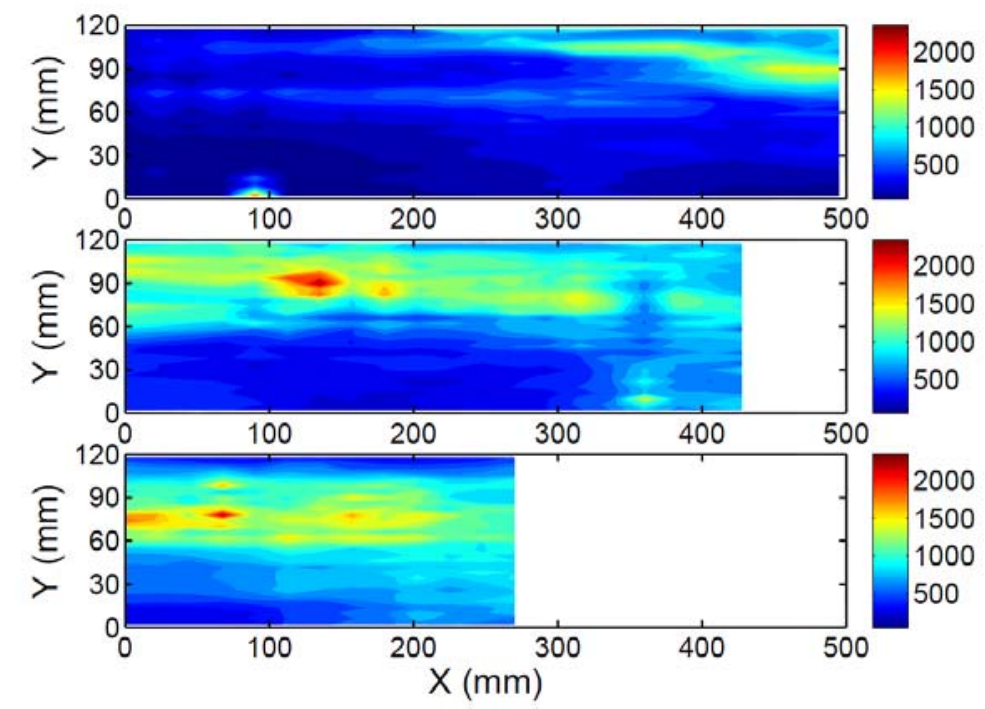

Figure 7: 2D contours of the spatial variability of the transverse elastic modulus in REBs

\begin{tabular}{llll}
\hline Board number & $\begin{array}{l}\text { Longitudinal variability } \\
\text { coefficient }(\mathrm{MPa} / \mathrm{mm})\end{array}$ & $\begin{array}{l}\text { Transverse variability } \\
\text { coefficient }(\mathrm{MPa} / \mathrm{mm})\end{array}$ & Anisotropy ratio \\
\hline 1 & 1.17 & 7.24 & 6.19 \\
2 & 1.61 & 8.87 & 5.51 \\
3 & 1.56 & 10.0 & 6.41 \\
\hline
\end{tabular}

Table 2: Longitudinal variability coefficients, transverse variability coefficients and anisotropy ratios in REBs. 


\section{EFFECT OF TIMBER MESOSTRUCTURE ON LOCAL ELASTIC MODULUS}

Typical examples of the correspondence between the local elastic modulus and the local mesostructure of spruce wood are given in Fig. 8, considering three specimens of $120 \mathrm{~mm}$ length. In specimen the TT-120-16-11-REB1 starting from the left, the grain angle, defined as the angle between the latewood and the vertical axis in Fig. 8, decreases up to $70 \mathrm{~mm}$ of the length. This is associated by a gradual increase in the local elastic modulus. The angle increases from $70 \mathrm{~mm}$ to $95 \mathrm{~mm}$ and after that decreases again, causing the local modulus to decrease and then increase, as a general trend. The maximum value happened to be at end of the specimen, where the grain angle has its lowest value. There is also a localized decrease in the value of the elastic modulus in the part of the specimen between $110 \mathrm{~mm}$ and $115 \mathrm{~mm}$ which is attributed to a decrease in the thickness of the latewood strips in this zone.

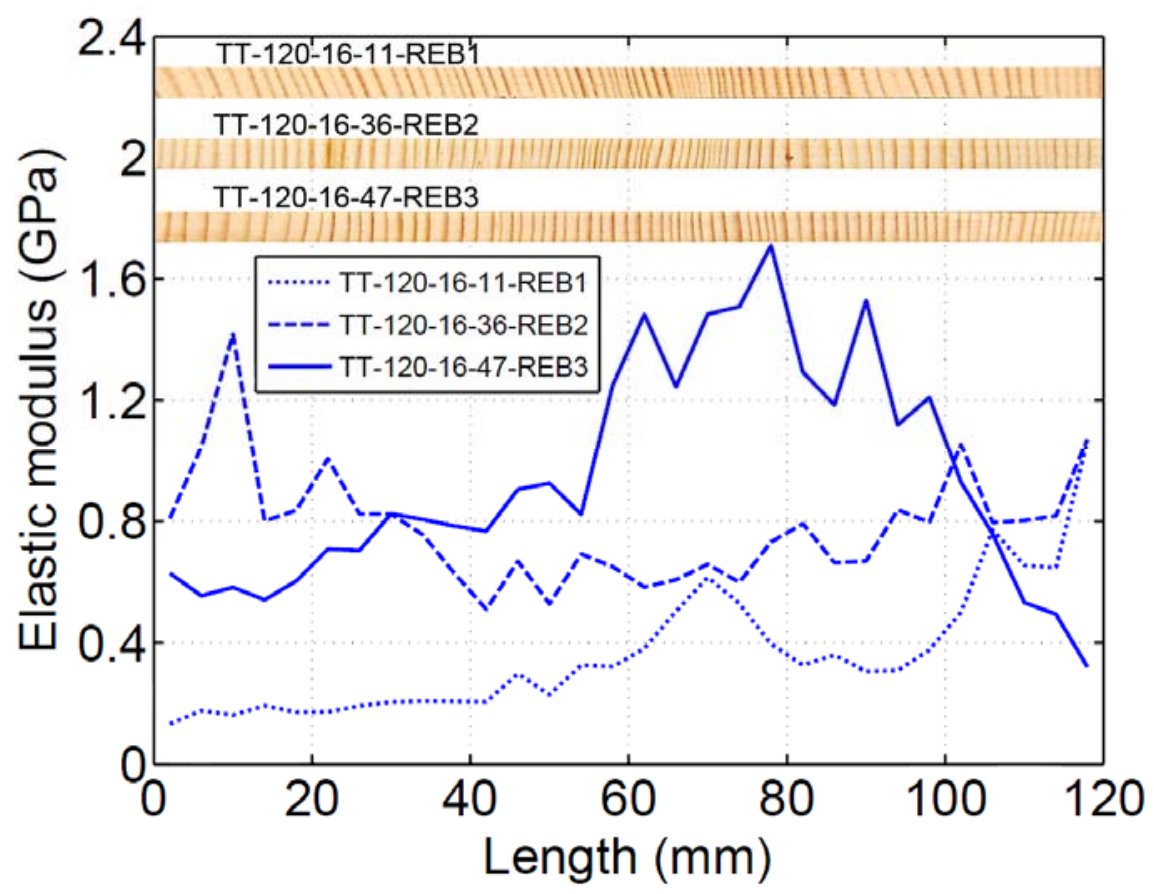

Figure 8: Correspondence between mesostructure of spruce and local transverse elastic modulus

In specimen the TT-120-16-36-REB2, over the first $10 \mathrm{~mm}$ of the specimen length, there is a decrease in the grain angle, then from $10 \mathrm{~mm}$ to about $60 \mathrm{~mm}$ the angle increases and after that the angle decreases. Correspondingly, the value of the elastic modulus first increases, then decreases and finally increases again, as a general trend. The effect of the grain angle on the local elastic modulus is even clear at the last $20 \mathrm{~mm}$ of the specimen length. In this segment of the specimen, the angle first increases a little and then decreases a little and, accordingly, there is a localized minimum in the value of the local elastic modulus in the middle of this segment. Also, a local maximum for the local modulus has happened in about $80 \mathrm{~mm}$ from the left end of the specimen. The grain angle has a local minimum in this position of the specimen length.

The local elastic modulus in specimen TT-120-16-47-REB3 has generally higher values compared the other two discussed specimens. The main reason is that, in this specimen, the grain angle has generally lower values. Only, in the first $30 \mathrm{~mm}$ and last $20 \mathrm{~mm}$ of the specimen length where the grain angle has higher values, the value of the modulus of this specimen is less than those of the other specimens. Also, in this specimen, latewood strips are thicker. 
The presence of higher volume fractions of latewood tends to increase the value of the local elastic modulus. This can either happen by presence of a higher number of latewood strips or thicker latewood strips. For example, in both specimens TT-120-16-11-REB1 and TT-12016-47-REB3 in the segment from $60 \mathrm{~mm}$ to $80 \mathrm{~mm}$ the number of latewood strips are higher than in the rest of segments in the corresponding specimens. Consequently, the transverse elastic modulus has a local peak in those segments.

\section{CONCLUSIONS}

- A highly significant spatial variability was observed in the transverse tensile elastic modulus of clear spruce wood. A difference of more than $1000 \%$ for the local elastic modulus was observed within some specimens.

- The variability of the transverse modulus is higher in the transverse direction than its variability in the longitudinal direction.

- The main reason for the spatial variability of the local elastic modulus is irregular changes in the mesostructure of the wood. The change in the grain angle is the most important factor influencing the local elastic modulus.

- Knots can substantially increase the transverse modulus of the clear wood around them.

\section{REFERENCES}

[1] J.D. Barrett, Effect of size on tension perpendicular-to-grain strength of Douglas-fir. Wood and Fiber, 6, 126-143, 1974.

[2] M. Vořechovský, Interplay of size effects in concrete specimens under tension studied via computational stochastic fracture mechanics. International Journal of Solids and Structures, 44, 2715-2731, 2007.

[3] S. Sriramula, M.K. Chryssanthopoulos, An experimental characterisation of spatial variability in GFRP composite panels. Structural Safety, 42, 1-11, 2013.

[4] J. Xavier, S. Avril, F. Pierron, J. Morais, Variation of transverse and shear stiffness properties of wood in a tree. Composites: Part A, 40, 1953-1960, 2009.

[5] J. Pereira, J. Xavier, J. Morais, J. Lousada, Assessing wood quality by spatial variation of elastic properties within the stem: Case study of Pinus pinaster in the transverse plane. Canadian Journal of Forest Research, 44, 107-117, 2014.

[6] R. Brandner, G. Schickhofer, Spatial correlation of tensile perpendicular to grain properties in Norway spruce timber. Wood Science and Technology, 48, 337-352, 2014.

[7] P.L. Clouston, F. Lam, A stochastic plasticity approach to strength modeling of strandbased wood composites. Composites Science and Technology, 62, 1381-1395, 2002.

[8] P.L. Clouston, F. Lam, Computational modeling of strand-based wood composites. Journal of Engineering Mechanics, 127, 844-851, 2001.

[9] T. Tannert, F. Lam, T. Vallée, Strength prediction for rounded dovetail connections considering size effects. Journal of Engineering Mechanics, 136, 358-366, 2010.

[10] Forest Product Laboratory, Wood handbook: Wood as an engineering material. USDA, Madison, Wis, 1999. 